\title{
OPEN Impact of reduced uterine perfusion pressure model of preeclampsia on metabolism of placenta, maternal and fetal hearts
}

\author{
Lana McClements ${ }^{1}$, Claire Richards ${ }^{1}$, Nikayla Patel ${ }^{2}$, Hao Chen ${ }^{1}$, Kimberly Sesperez ${ }^{1}$, \\ Kristen J. Bubb ${ }^{3}$, Anja Karlstaedt ${ }^{4}$ \& Dunja Aksentijevic ${ }^{2 \rrbracket}$
}

Preeclampsia is a cardiovascular pregnancy complication characterised by new onset hypertension and organ damage or intrauterine growth restriction. It is one of the leading causes of maternal and fetal mortality in pregnancy globally. Short of pre-term delivery of the fetus and placenta, treatment options are limited. Consequently, preeclampsia leads to increased cardiovascular disease risk in both mothers and offspring later in life. Here we aim to examine the impact of the reduced uterine perfusion pressure (RUPP) rat model of preeclampsia on the maternal cardiovascular system, placental and fetal heart metabolism. The surgical RUPP model was induced in pregnant rats by applying silver clips around the aorta and uterine arteries on gestational day 14, resulting in 40\% uterine blood flow reduction. The experiment was terminated on gestational day 19 and metabolomic profile of placentae, maternal and fetal hearts analysed using high-resolution ${ }^{1} \mathrm{H}$ NMR spectroscopy. Impairment of uterine perfusion in RUPP rats caused placental and cardiac hypoxia and a series of metabolic adaptations: altered energetics, carbohydrate, lipid and amino acid metabolism of placentae and maternal hearts. Comparatively, the fetal metabolic phenotype was mildly affected. Nevertheless, long-term effects of these changes in both mothers and the offspring should be investigated further in the future.

Cardiovascular disease is the main cause of death worldwide. Converging lines of evidence suggest that an adverse intrauterine environment precipitates maternal, placental and fetal adaptations, resulting in the developmental programming of cardiometabolic dysfunction in juvenile and adult offspring ${ }^{1-3}$. Preeclampsia is a cardiovascular pregnancy complication that occurs in $5-10 \%$ of pregnancies and is one of the leading causes of maternal and fetal morbidity and mortality worldwide ${ }^{4}$. It is associated with poor pregnancy outcomes and can lead to lifelong risk from cardiovascular complications in the offspring: an early onset of cardiac hypertrophy, aortic remodelling ${ }^{5-8}$, oxidative stress, endothelial dysfunction, hypertension, and adverse ischemia-reperfusion (IR) outcomes in adult life ${ }^{2}$. Compared to normotensive pregnancies, mothers with early-onset preeclampsia presenting prior to 34 weeks of gestation (EOPE) have increased mortality risk (up to 9-10-fold) from cardiovascular disease, whereas mothers diagnosed with late-onset preeclampsia after 34 weeks gestation (LOPE) are at a twofold increased risk of CVD-related mortality ${ }^{9,10}$. Recently, multiple common features were recognized between preeclampsia and heart failure with preserved ejection fraction (HFpEF) including factors such as hypertrophy, angiogenesis, inflammation and haemostasis ${ }^{11,12}$. However, the exact metabolic adaptations, which may impact in utero development and may lead to cardiometabolic consequences in adult life, have not been fully elucidated. Moreover, effective therapeutic and diagnostic strategies are still missing due to the lack of mechanistic insight into the causal nexus of preeclampsia. Nevertheless, an inability of the maternal cardiovascular system to alleviate preeclampsia-induced changes appears to have a critical role in defective placental development including oxidative stress, abnormal angiogenesis and pro-inflammatory responses ${ }^{13}$.

Pre-clinical models of preeclampsia are challenging to establish, due to the presence of inter-species variations and the fact that most animals do not develop spontaneous preeclampsia ${ }^{14}$. The most reliable animal

\footnotetext{
${ }^{1}$ School of Life Sciences, Faculty of Science, University of Technology Sydney, Sydney, NSW, Australia. ${ }^{2}$ Centre for Biochemical Pharmacology, William Harvey Research Institute, Barts and the London School of Medicine and Dentistry, Queen Mary University of London, Charterhouse Square, London EC1M 6BO, UK. ${ }^{3}$ Biomedical Discovery Institute, Monash University, Melbourne, Australia. ${ }^{4}$ Department of Cardiology, Smidt Heart Institute, Cedars Sinai

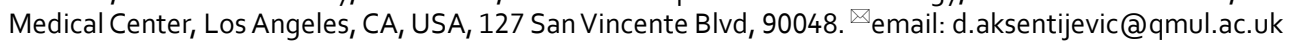




\begin{tabular}{|c|c|c|c|}
\hline & Sham $(n \geq 6)$ & RUPP $(n \geq 4)$ & $P$ value \\
\hline Maternal body weight (before surgery), g & $338.6 \pm 17.5$ & $379.5 \pm 4.87$ & 0.084 \\
\hline Maternal heart weight, $g$ & $1.02 \pm 0.05$ & $1.22 \pm 0.01$ & $0.008^{* *}$ \\
\hline Heart: Body weight, \% & $0.304 \pm 0.01$ & $0.323 \pm 0.005$ & 0.303 \\
\hline Embryo resorption (\%) & $2.38 \pm 1.51$ & $11.22 \pm 3.06$ & $0.04^{*}$ \\
\hline Embryo weight, g & $1.68 \pm 0.025$ & $1.61 \pm 0.02$ & 0.04 \\
\hline Placental weight, $g$ & $0.41 \pm 0.009$ & $0.39 \pm 0.006$ & 0.047 \\
\hline Heart rate, bpm & $369.3 \pm 4.8$ & $398.8 \pm 3.7$ & $0.0006^{\star * *}$ \\
\hline Systolic BP, mmHg & $112.6 \pm 1.3$ & $127.8 \pm 1.9$ & $<0.0001^{* * *}$ \\
\hline Diastolic BP, mmHg & $87.6 \pm 1.7$ & $104.0 \pm 1.8$ & $<0.0001^{* * *}$ \\
\hline MABP, $\mathrm{mmHg}$ & $100.9 \pm 1.5$ & $116.7 \pm 1.7$ & $<0.0001^{* * *}$ \\
\hline Stroke volume, $\mu \mathrm{l}$ & $215.0 \pm 4.326$ & $230.0 \pm 13.26$ & 0.304 \\
\hline Cardiac output, $\mathrm{mL} / \mathrm{min}$ & $79 \pm 3$ & $85 \pm 5$ & 0.067 \\
\hline Ejection fraction, $\%$ & $82 \pm 2$ & $78 \pm 2$ & 0.233 \\
\hline Fractional shortening, $\%$ & $52 \pm 2$ & $49 \pm 2$ & 0.236 \\
\hline Corrected LV mass, mg & $600 \pm 14$ & $706 \pm 68$ & $0.037^{\star}$ \\
\hline $\mathrm{LV}$ anterior wall systolic, $\mathrm{mm}$ & $2.9 \pm 0.12$ & $2.9 \pm 0.11$ & 0.672 \\
\hline $\mathrm{LV}$ anterior wall diastolic, $\mathrm{mm}$ & $1.5 \pm 0.04$ & $1.8 \pm 0.17$ & 0.152 \\
\hline LV posterior wall systolic, $\mathrm{mm}$ & $2.8 \pm 0.14$ & $2.9 \pm 0.09$ & 0.303 \\
\hline LV posterior wall diastolic, $\mathrm{mm}$ & $1.6 \pm 0.05$ & $1.6 \pm 0.11$ & 0.994 \\
\hline
\end{tabular}

Table 1. Morphological features and cardiac function characteristics of the RUPP model (maternal and fetal). Unpaired Student's t-test or Mann-Whitney test were used depending on data distribution. This figure was originally published in Biology of Sex Differences and used with permission ${ }^{26}$ (https://doi.org/10.1186/s13293021-00376-1). BP blood pressure, $L V$ left ventricular, $M A B P$ mean arterial blood pressure, $R U P P$ reduced uterine perfusion pressure. ${ }^{\star} P<0.05,{ }^{* * *} P<0.001 .{ }^{* *} P<0.01$

model of preeclampsia is the surgically-induced reduced uterine perfusion pressure (RUPP) model. This model has been shown to induce hypertension ${ }^{15}$, proteinuria ${ }^{15}$, renal dysfunction ${ }^{15,16}$, an anti-angiogenic state ${ }^{17}$, inflammation ${ }^{18-20}$, vasoconstriction ${ }^{21}$, oxidative stress ${ }^{22}$, cardiac dysfunction ${ }^{23}$ and intrauterine growth restriction (IUGR) $)^{24,25}$ like that of preeclampsia in humans.

We have also recently shown in this model increased cardiac expression of the anti-angiogenic protein, FKBPL, and the presence of placental and cardiac fibrosis ${ }^{26}$. Given the significant association between preeclampsia and CVD, a more thorough investigation of cardiac health in the RUPP model is required. In this study, we demonstrate in the RUPP rat model of preeclampsia, the presence of cardiovascular and metabolic remodelling of maternal heart and placenta. We also show that the series of functional and metabolic maternal adaptations to early restricted angiogenesis precede metabolic alterations in the fetal heart.

\section{Methods}

Reduced uterine perfusion pressure pregnancy model in rats. Time-mated pregnant SpragueDawley rats were purchased from the Animal Resources Centre (ARC) and were fed a standard sterile chow diet with water access ad libitum. On gestational day (GD) 14, pregnant rats were randomised to the Sham (control; $\mathrm{n}=8)$ or RUPP $(\mathrm{n}=7)$ procedure as previously described ${ }^{15,26,27}$. Briefly, silver clips were applied to the aorta above the iliac bifurcation $(0.203 \mathrm{~mm}$ ID) and both the right and left uterine arcades $(0.100 \mathrm{~mm}$ ID) to reduce the blood flow to the uterus by $\sim 40 \%$ in the RUPP group whereas rats in the Sham (control) group underwent a similar procedure, without clips being applied. On GD19, after clinical phenotyping was completed, rats were euthanized as per local standard operating procedures using isoflurane overdose/exsanguination followed by removal of the heart. Maternal hearts, placentae and embryo hearts were collected, weighed and snap frozen in liquid $\mathrm{N}_{2}$. All clinical phenotyping, placental and embryo data for these rats were reflective of the preeclampsia phenotype as published previously ${ }^{26}$ and reproduced here with permission (Table 1). For this study, five matching maternal hearts, placenta and fetal hearts were available from each group for downstream metabolomic analysis as described below. All animal experiments were approved by the Northern Sydney Local Health District Animal Ethics Committee (Animal Ethics number: RESP/18/317) and carried out in accordance to the Australian code for the care and use of animals for scientific purposes and ARRIVE guidelines.

${ }^{1} \mathrm{H}$ Nuclear magnetic resonance spectroscopy metabolomic profiling. Frozen, weighed and pulverized hearts (fetal and maternal) and placentae were subjected to methanol/water/chloroform dualphase extraction ${ }^{28}$. The aqueous upper phase was separated from the chloroform and protein fractions. $30 \mathrm{mg}$ chelex-100 was added to chelate paramagnetic ions, vortexed and centrifuged at $3600 \mathrm{RPM}$ for $5 \mathrm{~min}$ at $4{ }^{\circ} \mathrm{C}$. The supernatant was added to a fresh Falcon tube containing $10 \mu \mathrm{L}$ universal $\mathrm{pH}$ indicator solution followed by vortexing and lyophilisation. Dual-phase-extracted metabolites were reconstituted in $600 \mu \mathrm{L}$ deuterium oxide (containing $8 \mathrm{~g} / \mathrm{L} \mathrm{NaCl}, 0.2 \mathrm{~g} / \mathrm{L} \mathrm{KCl}, 1.15 \mathrm{~g} / \mathrm{L} \mathrm{Na} 2 \mathrm{HPO} 4,0.2 \mathrm{~g} / \mathrm{L} \mathrm{KH} 2 \mathrm{PO} 4$ and $0.0075 \% \mathrm{w} / \mathrm{v}$ trimethylsilyl propanoic acid (TSP)) and adjusted to $\mathrm{pH} \approx 6.5$ using $1 \mathrm{M}$ hydrochloric acid and/or $1 \mathrm{M}$ sodium hydroxide ${ }^{28}$. 
Samples were analysed using a vertical-bore, ultra-shielded Bruker 14.1.T (600 MHz) spectrometer with a bbo probe at $303 \mathrm{~K}$. Spectra were acquired with the Bruker noesygppr1d pulse sequence with 128 scans, 4 dummy scans and $20 \mathrm{ppm}$ sweep width, acquisition time of $2.6 \mathrm{~s}$, pre-scan delay of $4 \mathrm{~s}, 90^{\circ}$ flip angle and experiment duration of $14.4 \mathrm{~min}$. TopSpin (version 4.0.5) software was used for data acquisition and for metabolite quantification. FIDs were multiplied by a line broadening factor of $0.3 \mathrm{~Hz}$ and Fourier-transformed, phase and automatic baseline-correction were applied. Chemical shifts were normalized by setting the TSP signal to $0 \mathrm{ppm}$. In terms of glycogen quantification, given that it is a large macromolecule containing multiple glucose monomers, we measured a number of mobile ${ }^{1} \mathrm{H}$ in the glucose monomers that are present in the NMR peak, normalized to the reference.

Peaks of interest were integrated automatically using a pre-written integration region text file and then manually adjusted where required. Assignment of metabolites to their respective peaks was carried out based on previously obtained in-house data, confirmed by chemical shift and using Chenomx NMR Profiler Version 8.1 (Chenomx, Canada). Peak areas were normalized to the TSP peaks and metabolite concentrations were quantified per gram tissue wet weight ${ }^{29,30}$.

Real-time quantitative polymerase chain reaction (RT-qPCR). Relative mRNA expression of galectin-3 in the placentae in Sham and RUPP rats was measured by RT-qPCR. Frozen tissue was homogenised with TRIsure reagent (Bioline, Australia) and isolated according to the manufacturer's protocol. Once purified, the RNA was reverse transcribed to cDNA using a Tetro cDNA synthesis kit (Bioline, Australia) and relevant primers (Supplemental Table S1). RT-qPCR was performed using a SensiFAST SYBR No-ROX kit (Bioline, Australia) according to the manufacturer's protocols and CFX96 Real-Time System thermal cycler (Bio-Rad, United States). The mRNA expression levels were normalized to those of $\beta$-actin and transformed by the $\Delta \Delta \mathrm{CT}$ method.

Western blotting. Protein lysates were generated from frozen tissue samples by homogenising with RIPA lysis buffer. Protein was quantified by BCA assay (Thermo Fisher, United States) prior to separation by SDSPAGE. Following transfer, the membranes were imaged for total protein using the Stain Free imaging component of the ChemiDoc imaging system (Bio-Rad, United States) and blocked with $5 \%$ skimmed milk. The membranes were probed with mouse anti-HIF1- $\alpha$ monoclonal antibody (BD Bioscience, United States) and corresponding sheep HRP-linked anti-mouse IgG secondary antibody (GE Healthcare, United Kingdom). Immunoreactive bands were visualised by reacting with Clarity Western ECL (Bio-Rad, United States) and imaged using the ChemiDoc. Relative HIF1- $\alpha$ protein expression was normalized to the intensity of bands detected in the stain free image of each blot as a loading control.

Metabolic network analysis of placenta. Given that the most extensive metabolite concentration changes were identified in placenta ( $>2$ metabolites), network plots of metabolite co-regulation were generated with the igraph package in $\mathrm{R}$ programming language (4.1.0). Each node was representative of a significantly different metabolite from different tissues in RUPP mice compared to the sham controls. Only the strongly correlated nodes were shown (absolute Pearson correlation coefficient $|\mathrm{r}|>0.7$ ), and colour of each edge was indicative of Pearson $r$. The functional classifications of nodes were annotated as the colour of nodes. Each node was sized proportionally to the epigenvector centrality (the importance of the network).

In Silico modelling. In silico simulations (maternal hearts) were conducted using the metabolic network of the cardiomyocyte CardioNet ${ }^{31,32}$ as described in the Supplementary Information. Details of all reactions and their metabolic subsystems were annotated based on the Kyoto Encyclopedia of Genes and Genomes database ${ }^{33}$.

Statistical analysis. Statistical analysis was performed in GraphPad Prism (v9). Normality of data distribution was examined using Shapiro-Wilk's normality test. Sham and RUPP groups were compared using the unpaired student's t-test (normally distributed data) and Mann-Whitney U test (non-normally distributed data) with statistically significant results equivalent to a p-value of 0.05 or less.

\section{Results}

The pre-clinical rat model of preeclampsia resulting from the RUPP surgical intervention on gestational day 14 led to a series of maternal and fetal physiological adaptations (Table 1). We have recently shown that the RUPP procedure leads to reduced placenta and fetal weight as well as increased fetal resorption (Table 1$)^{26}$. Furthermore, it triggers cardiac and placental fibrosis, maternal haemodynamic overload resulting in increased heart rate, systolic, diastolic and mean arterial pressures collectively resulting in increased LV mass and cardiac hypertrophy indicative of diastolic dysfunction (Table 1$)^{26}$. However, there was no evidence of maternal cardiac failure as in vivo cardiac function parameters including ejection fraction, cardiac output and stroke volume were comparable to sham controls ${ }^{26}$. These observations are collectively reflective of preeclampsia in humans. Furthermore, RUPP intervention leads to placental hypoxia indicated by increase in hypoxia inducible factor (HIF)-1 $\alpha$ protein expression (Fig. $1 ; P<0.05$ ). However, there were no changes in the mRNA expression of the inflammation marker galactin-3 in maternal tissues (placenta and heart, Supplementary Fig. S1).

RUPP impact on placental, maternal and fetal cardiac metabolism. Using high resolution ${ }^{1} \mathrm{H}$ NMR spectroscopy (representative spectra shown in Fig. 2) we were able to identify a series of changes in the metabolomic profiles of maternal hearts, fetal hearts and placentae due to RUPP-induced preeclampsia. Metabolomic profile of maternal hearts show reduced NAD/NADH ratio indicative of an altered redox pool (Fig. 3A), 
A

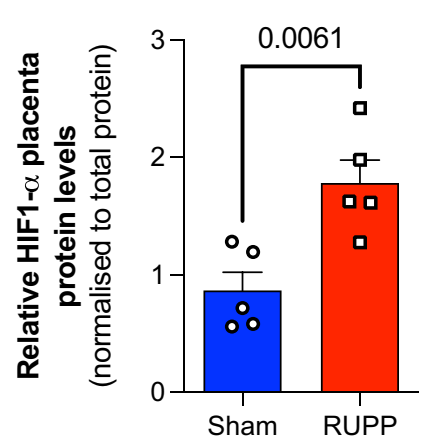

B

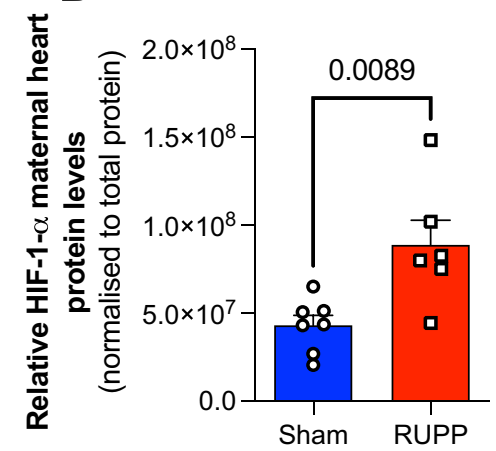

Figure 1. RUPP increases HIF1 $\alpha$ expression in placenta and maternal hearts. RUPP leads to increase in HIF-1 $\alpha$ protein expression in placenta (A) and maternal hearts (B). Protein lysates were extracted from placenta and heart from each rat and western blotting performed to determine the expression of HIF-1 $\alpha$ relative to the total protein. Two group comparison was carried out using the student $\mathrm{t}$ test, $\mathrm{n} \geq 5$; $P$ values $<0.05$ shown in individual plots.

increased glucose and glycogen levels (Fig. 3C) as well as altered lipid metabolism constituents, acetate and acetyl carnitine (Fig. 3D). Furthermore, there were decreased levels of succinate (Fig. 3B) however given that the succinate/fumarate ratio was unaltered (Sham $26 \pm 8$ vs RUPP $15 \pm 5, P<0.2$ ), the overall TCA cycle flux appears unaltered. Despite a series of changes in metabolite levels across different metabolic compartments, there is no evidence of energetic deficit or stress as creatine reserve and PCr/ATP ratio are comparable to sham controls. Furthermore, there is no change in the total adenine nucleotide (TAN) pool as ATP, ADP and AMP levels are comparable between the groups (Fig. 3A) and no alterations in the amino acid metabolism short of aspartate (Fig. 3E). Furthermore, there is evidence that preeclampsia induced maternal cardiac hypoxia as the protein levels of HIF-1 $\alpha$ are increased compared to the sham controls (Fig. 1B) in agreement with the altered carbohydrate pool (Fig. 3C).

Placentae of the RUPP dams developed various metabolic adaptations. The RUPP placenta is characterized by altered TAN pool and reduced PCr/ATP ratio energy reserve (Fig. 4A), without changes in the TCA cycle (Fig. 4B). Furthermore, there are alterations in carbohydrate, lipid and amino acid metabolism: enhanced glycogen content (Fig. 4C), increased carnitine, choline and phosphocholine levels (Fig. 4D) as well as a decrease in the levels of the amino acid valine (Fig. 4E). Reactome metabolic network analysis identified that ATP (redox and energetics) and phosphocholine (lipid metabolism) were the most influential metabolites in the placenta metabolic network (Supplementary Fig. S2).

Remarkably, despite maternal cardiovascular remodelling including increased LV mass, cardiac hypertension ${ }^{26}$ and placental metabolic alterations, metabolism of the fetal hearts (Fig. 5A-E) remained unaltered as the metabolomic profile was largely comparable between RUPP and control pregnancies short of a modest reduction in glycine levels (Fig. 5E). However, of notable change is the $51 \%$ reduction in fetal myocardial glucose levels (Fig. 5C).

Computational modelling identifies metabolic flux adaptations in the RUPP maternal hearts. To assess the impact of the RUPP model of preeclampsia on maternal cardiac metabolism at a systems scale, we conducted mathematical modelling using CardioNet ${ }^{31,32}$. CardioNet has been successfully applied to identify limiting metabolic processes and estimate flux distributions ${ }^{34-37}$. We determined flux distributions using flux balance analysis (FBA), which seeks to estimate flux rates during steady-state while optimising an objective function (see Supplementary Methods for details). The optimisation problem was to maximize ATP provision within a set of constraints defined by our experimental conditions. Maternal heart metabolic data measured by ${ }^{1} \mathrm{H}$ NMR spectroscopy was included into the simulations to determine which metabolic flux distributions are consistent with the maternal cardiac adaptation during preeclampsia. These simulations were conducted without constraining enzymatic activities; thus, the optimisation problem was defined by experimentally determined metabolite levels. Principal component analysis (PCA) (Supplementary Fig. S3A) of estimated flux distributions clearly differentiated samples according to the presence of preeclampsia. Annotation enrichment of the observed clusters and unsupervised hierarchical cluster analysis (Supplementary Fig. S3B) demonstrate extensive metabolic flux remodelling in glycolysis and lipid metabolism resulting in maintained ATP provision in the maternal heart. In silico modelling shows that RUPP hearts require overall more nutrients to maintain ATP provision and are exposed to increased ROS stress. Preeclampsia enhances glucose uptake and increases the rate of glycolysis as the key reactions involved in glycolysis were upregulated (Fig. 6) accompanied by increased lactate efflux (Fig. 6). Thus overall, Cardionet modelling indicates increased carbohydrate use. However, carbons from glycolysis are still predicted to enter the Krebs Cycle for oxidative phosphorylation. Furthermore, in silico modelling indicates that in RUPP conditions endogenous lipid pools are utilized to provide ATP. This increases oxidation of fatty acids (hexadecenoyl-CoA, stearoyl-CoA, palmitoyl-CoA, oleoyl-CoA) derived from phospholipids and is potentially responsible for the observed increase in the production of reactive oxygen species (Fig. 6). 


\section{Maternal heart}

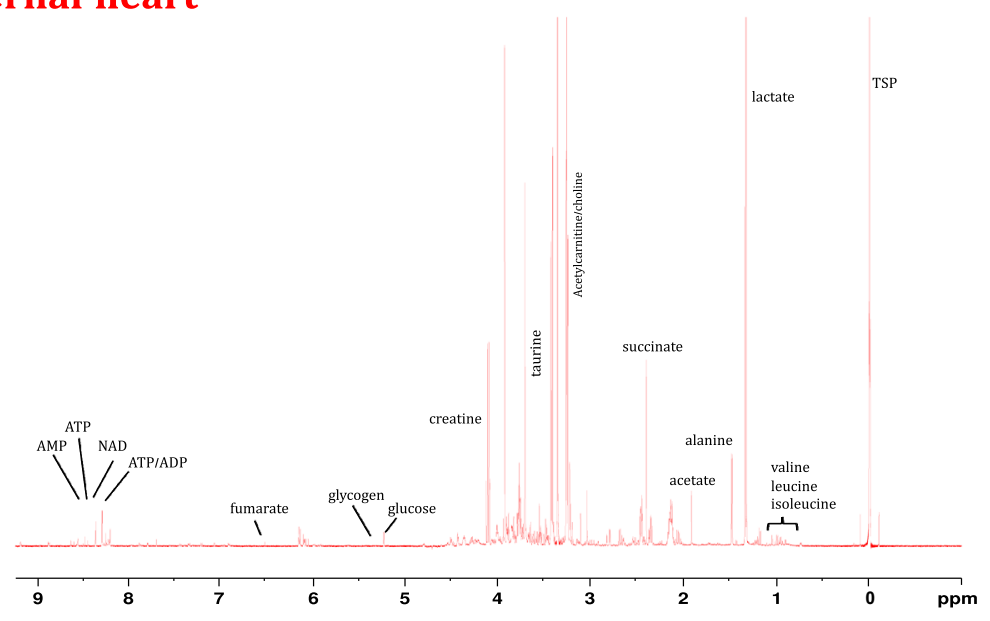

\section{Fetal heart}
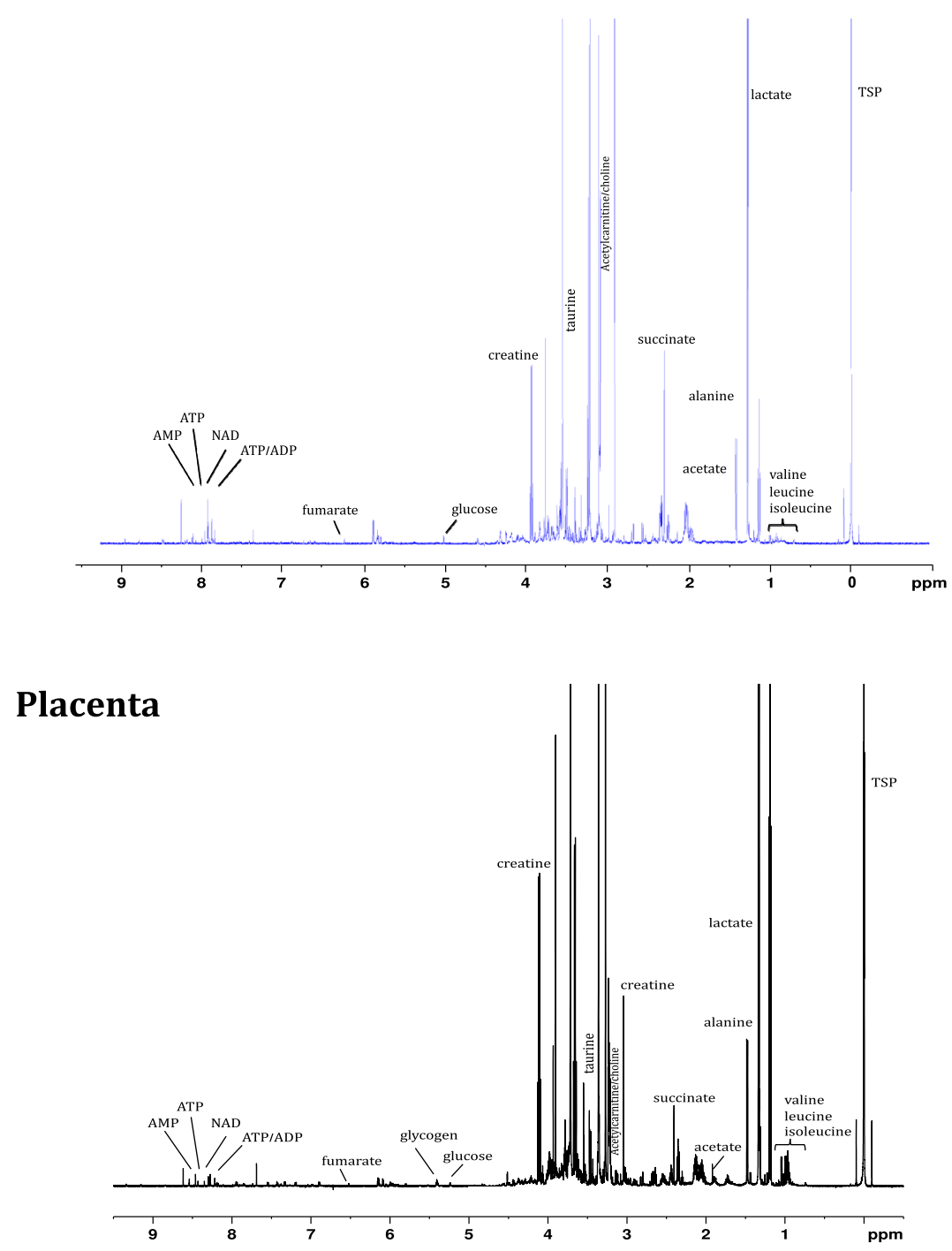

Figure 2. Representative ${ }^{1} \mathrm{H}$ NMR spectra from heart (maternal and fetal) and placenta. 


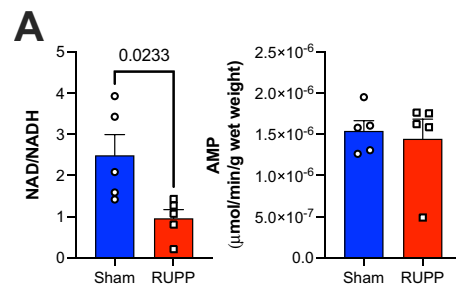

B
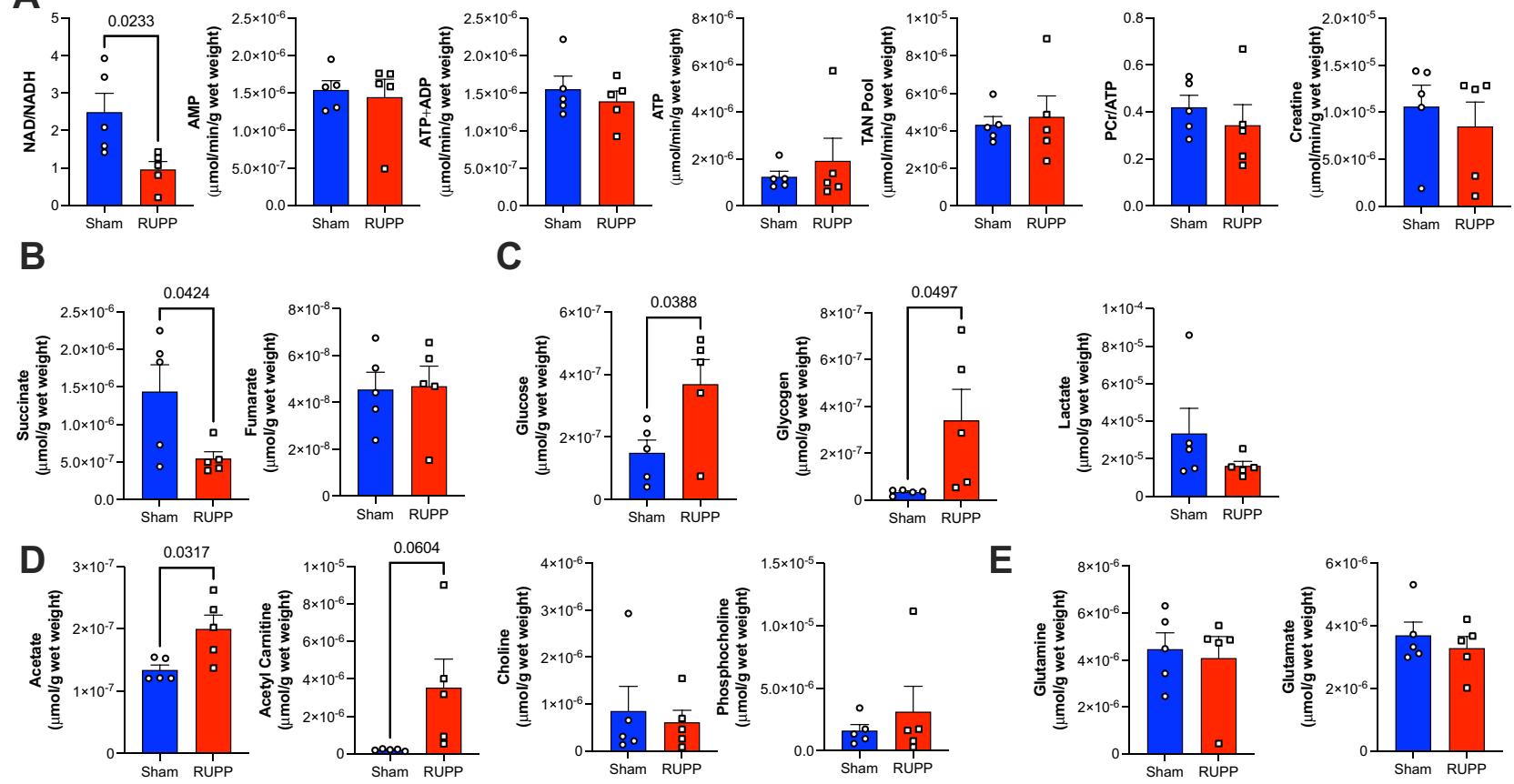

C
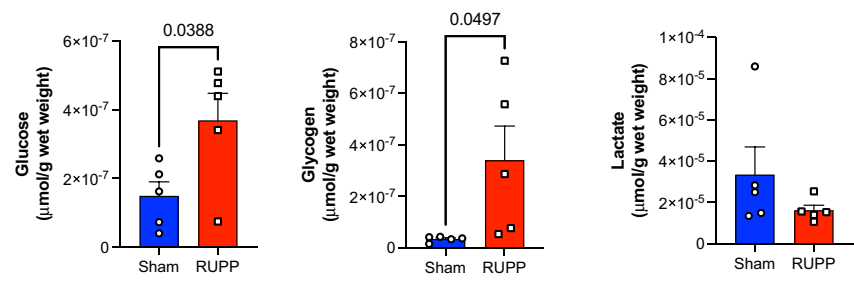

E
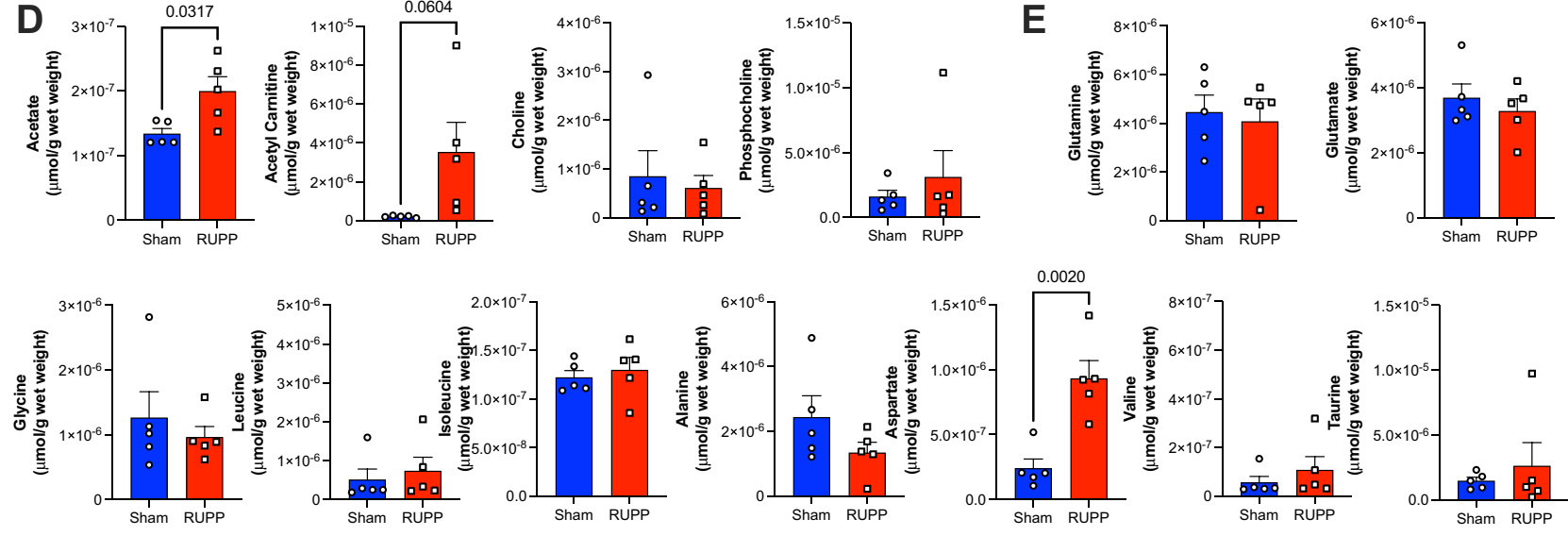

Figure 3. Metabolomic profile of RUPP model of maternal hearts. Metabolite concentrations are grouped into (A) Redox and Energetics (B) TCA cycle (C) Carbohydrate metabolism (D) Lipid metabolism (E) Amino acid metabolism. Two group comparison was carried out using the student $\mathrm{T}$ test (normally distributed data) or Mann-Whitney U test (non-normally distributed data: acetate, aspartate, NADH, lactate, leucine, valine, lactate, glutamine, choline) $\mathrm{n}=5$ /group. TAN-total adenine nucleotide pool (AMP + ADP + ATP). PCrphosphocreatine $\mathrm{n} \geq 5$; $P$ values $<0.05$ shown in individual plots.

\section{Discussion}

In this study we have examined the impact of surgically induced reduced perfusion to the uterus in pregnant rats on the metabolism of placenta, maternal and fetal hearts. Cardiovascular alterations particularly cardiac metabolism, have not been extensively studied in this model of preeclampsia ${ }^{38}$. Reduced uterine perfusion leads to pathophysiological effects consistent with preeclampsia-increased fetal resorption, intrauterine growth restriction and increased haemodynamic load in maternal cardiovascular system resulting in increased heart rate, systolic, diastolic and mean arterial pressures resulting in cardiac hypertrophic enlargement ${ }^{26}$. Furthermore, our RUPP model is characterized by impaired placental, renal and cardiac phenotype ${ }^{26}$ in agreement with the other studies characterizing the RUPP mode $^{39,40}$. Given the well-established association between preeclampsia and increased risk of development of cardiovascular complications in the offspring ${ }^{41}$, this is an important aspect in characterizing the manifestations of pre-eclampsia. Of notice, our recently published study describes the overlapping mechanisms between preeclampsia and future cardiovascular disease with angiogenesis- and inflammatory-related pathways playing a key role ${ }^{12}$.

However, this is the first study that explores the maternal and fetal cardiometabolic health in the pre-clinical RUPP model of preeclampsia. Our study shows that systemic haemodynamic overload triggered by reduced uterine perfusion impacts metabolism of maternal heart and placenta. Resultant increase in HIF-1a expression corresponds to adaptive metabolic remodelling in response to RUPP ${ }^{42,43}$. Changes observed in RUPP maternal hearts are suggestive of adaptive metabolic remodelling as we see no evidence of energetic deficit with comparable high energy phosphates. Cardiac metabolic changes manifest as a drop in redox pool (NAD/NADH ratio) and enhanced carbohydrate metabolism in terms of uptake and storage of glucose. However, whilst the myocardial lactate concentration is comparable between the groups, our in silico modelling has shown enhanced lactate efflux in keeping with previously reported increased circulating lactate levels in RUPP models ${ }^{44,45}$. Furthermore, we have recently shown that altered glucose metabolism in pre-gestational or gestational diabetes results in increased risk of placental vascular dysfunction and malperfusion ${ }^{46}$. 
A

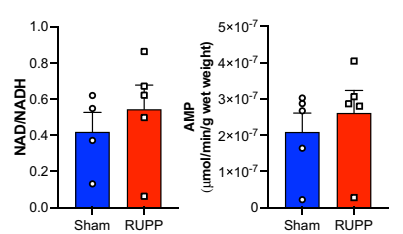

B
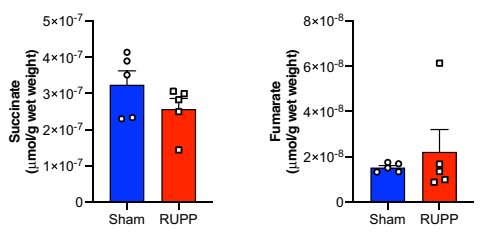

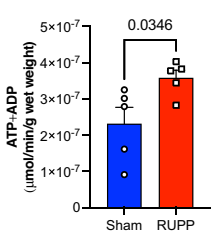

(

C
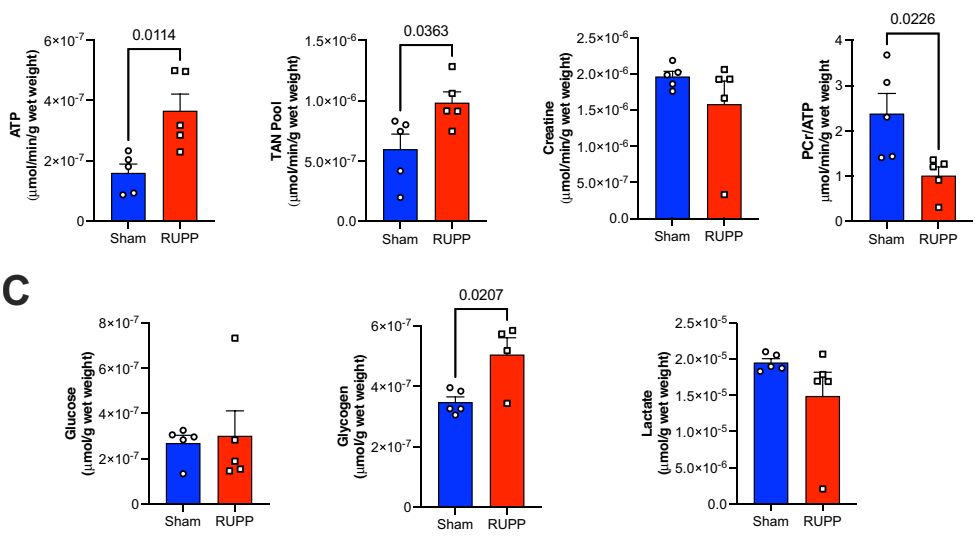

E
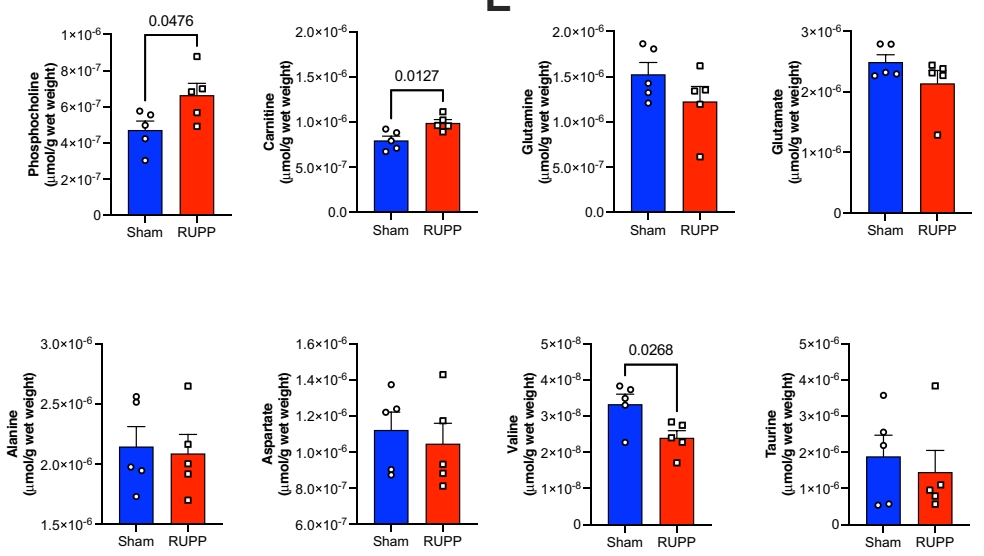

Figure 4. Impact of RUPP on placenta metabolism. Metabolite concentrations are grouped into (A) Redox and Energetics (B) TCA Cycle (C) Carbohydrate metabolism (D) Lipid metabolism (E) Amino acid metabolism. Two group comparison was carried out using the student $\mathrm{T}$ test (normally distributed data) or Mann-Whitney $\mathrm{U}$ test (non-normally distributed data: fumarate, leucine, isoleucine, lactate, glucose) TAN-total adenine nucleotide pool (AMP + ADP + ATP). $\mathrm{n}=5$ /group. $\mathrm{PCr}$-phosphocreatine. $P$ values $<0.05$ shown in individual plots.

Our data has shown that the RUPP model of preeclampsia induces a change in maternal myocardial substrate use concurrent with the presence of cardiac hypertrophy ${ }^{47}$. We observed increased levels of acetate and acetyl carnitine, potentially indicative of increased lipid utilisation. However, acetate accumulation observed in RUPP maternal hearts could also arise as the metabolic consequence of enhanced oxidative glucose metabolism and pyruvate dehydrogenase activity ${ }^{48}$. The RUPP metabolic profile was characterized by increased ATP demand met by increased glucose utilization but also enhanced use of endogenous lipids accompanied by increased production of reactive oxygen species (ROS). We speculate that the increased oxidation of endogenous lipids is responsible for increased ROS production. The in silico profile indicates that fatty acid oxidation is only feasible if oxygen consumption can simultaneously be increased. The model predicts that if oxygen becomes limiting, this would further increase the use of glucose by maternal RUPP hearts. The drop in the NAD $/$ NADH ratio is also indicative of the altered nicotinamide turnover, which has recently been shown to be an important hallmark of metabolic remodelling in cardiac pathologies ${ }^{49}$.

Expectedly, reduced uterine perfusion exhibited the strongest metabolic effect on placentae. A myriad of studies over the years have shown that the placenta is the fundamental source of fetal programming in adverse intrauterine environment and that metabolic remodelling is the incipient step in the progression to more severe forms of fetal growth restriction ${ }^{50}$. Reduced tissue perfusion normally triggers a range of responses including increased angiogenesis and erythropoiesis in order to improve $\mathrm{O}_{2}$ availability needed for cellular survival under hypoxic conditions. In both human and animal placentas, hypoxia-triggered responses result in increased density of villous capillaries and elevated secretion of angiogenic and hematopoietic factors (ie. VEGF, erythropoietin $)^{51-56}$. However, as our previous work has shown, preeclampsia is characterized by an anti-angiogenic phenotype driven by the upregulation in FKBPL expression or increase in sFlt-1, which would disrupt this primary hypoxia defence mechanism triggered by reduced perfusion ${ }^{57,58}$.

Increased ATP levels driving an increase in the TAN pool are indicative of altered placental ATP homeostasis driven by reduced perfusion and resultant hypoxia as evidenced by increased HIF-1 $1 \alpha$ expression. In nonplacenta tissue including hearts this would be a rare finding as ATP levels are tightly regulated (in part via the Randle cycle) to avoid energetic waste. This observation could potentially have two implications. First, RUPP 
A
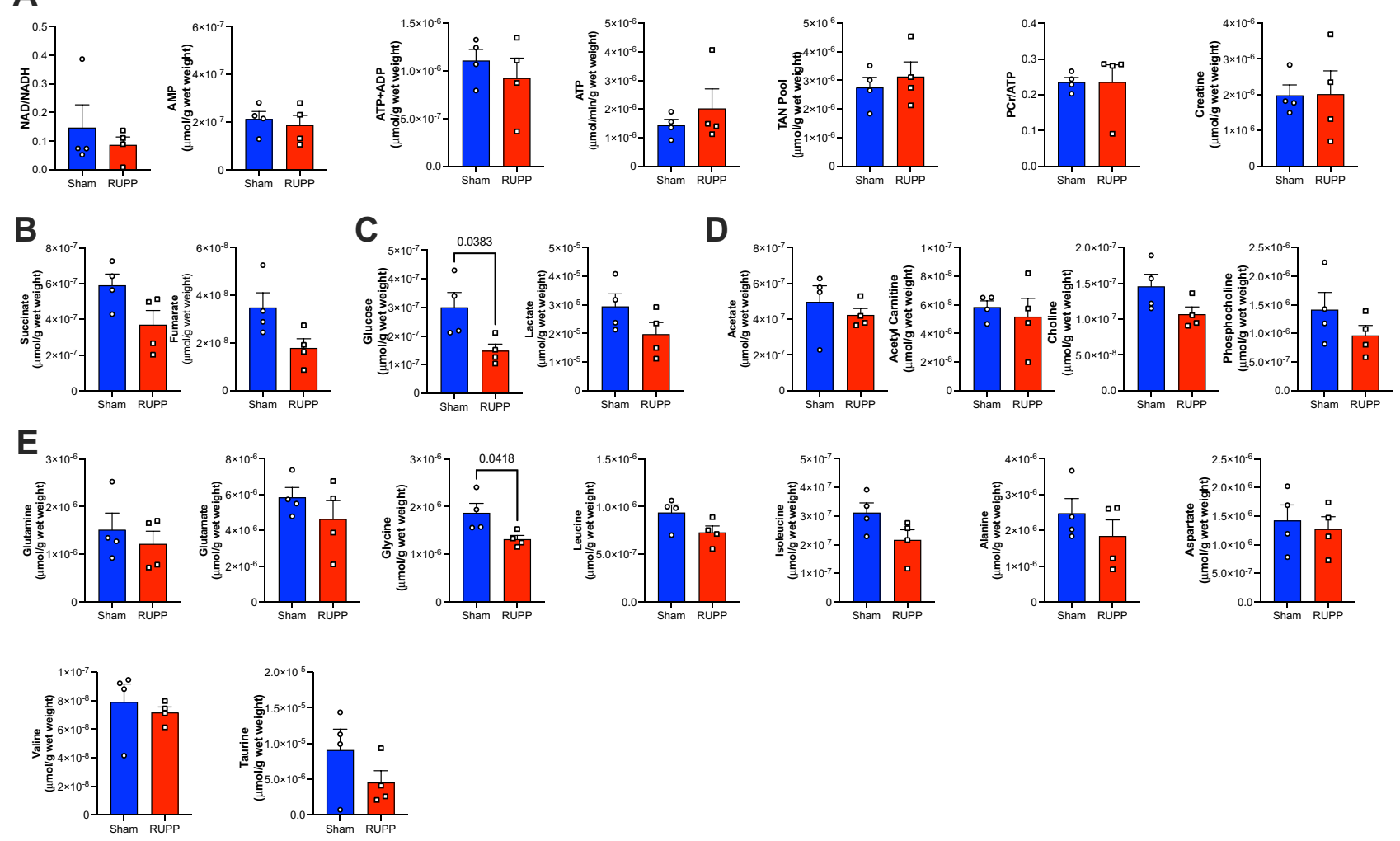

Figure 5. Impact of RUPP on fetal cardiac metabolism. Metabolite concentrations are grouped into (A) Redox and Energetics (B) TCA Cycle (C) Carbohydrate metabolism (D) Lipid metabolism (E) Amino acid metabolism. Two group comparison was carried out using the student $\mathrm{T}$ test (normally distributed data) or Mann-Whitney U test (non-normally distributed data: NAD/NADH, valine, ATP, Taurine, PCr/ATP) TANtotal adenine nucleotide pool (AMP + ADP + ATP). $n=4$ /group. $\mathrm{PCr}$-phosphocreatine. $P$ values $<0.05$ shown in individual plots.

placentae with an anti-angiogenic phenotype ${ }^{17}$ and altered HIF-1 $\alpha$ could lead to reduced energy demand by reducing the activity of ATP utilisers, thus reducing ATP use. This response in placental biology is broadly defined as "demand reduction" and consists of the attenuation of energy-requiring processes, non-essential to cellular survival, including gross energy demands such as fetal movement ${ }^{59}$. At the tissue level the inhibition of transcription, translation and related processes is dependent on protein synthesis, such as cellular growth and proliferation ${ }^{60}$. Furthermore, this interpretation would be entirely consistent with the traditional observation that hypoxia triggers adaptation to augment the ATP supply including via non-oxidative pathways. In the placenta this process has been demonstrated most clearly by an increase in glucose uptake and consumption as well as other key elements of the glycolytic metabolism ${ }^{50,61-65}$. Reduction in oxidative pathways, specifically a shift towards carbohydrate metabolism of RUPP placentae, would also be supported by the accumulation of carnitine due to altered fatty acid use.

Furthermore, we also observe changes in placental choline and phosphocholine levels, indicative of altered and enhanced nutrient transport in response to restricted vascularization and perfusion. Choline is an important bioactive micronutrient often grouped with the B vitamins. Placental transport of choline from the maternal circulation is largely mediated by the choline transporter-like protein 1 (CTL1) ${ }^{66}$. Within the placenta, choline and its metabolites perform crucial roles in placental and foetal development, especially the development of the brain. Enhanced choline uptake from maternal circulation, which would lead to enhanced levels in the placenta, would help to maintain both essential and non-essential amino acids for fetal development as well as enhance glycogen synthesis in the placenta as the additional source of energy ${ }^{67}$.

Thus, enhanced choline and its metabolite phosphocholine levels would also explain the increased glycogen content we observed in RUPP placentae. Whilst the metabolomic profile of amino acids was mostly unaffected by RUPP, levels of valine were markedly reduced suggesting enhanced fetal uptake and oxidation ${ }^{68}$.

Despite the series of metabolic adaptations in maternal hearts and placentae we observed in our study, these were unable to prevent intrauterine growth restrictions and fetal loss as RUPP dams had higher fetal resorption rate in agreement with both human preeclampsia and animal model outcomes. Although surviving embryos were smaller overall ${ }^{26}$, there is no evidence of extensive cardiometabolic remodelling. However, whilst there may not be many metabolite levels altered in the hearts of RUPP pregnancy fetuses, there is a marked reduction in the level of the most critical metabolite, glucose. Unlike adult hearts, fetal hearts rely on glucose as the main source of $\mathrm{ATP}^{47}$. Glucose is the principal energy substrate for the placenta and the fetus and is essential for normal fetal metabolism and growth. However, given that there are no alterations in fetal heart energy reserve, redox 

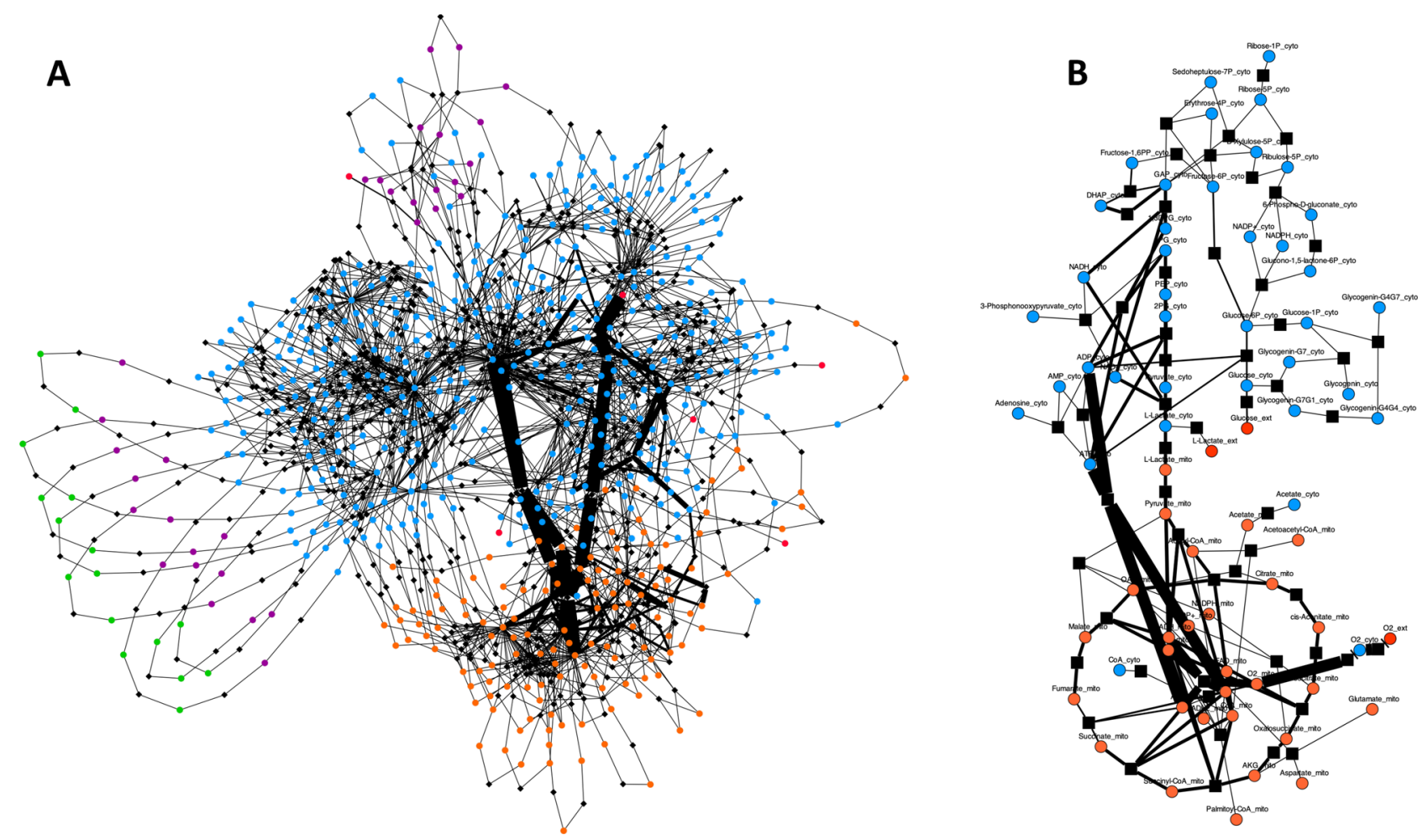

Figure 6. CardioNet in silico maternal heart metabolic flux changes in response to RUPP. Graph denotes estimated flux distribution in response to RUPP. The coloured nodes represent metabolites assigned to five different compartments: extracellular space, cytosol, mitochondria, microsome, lysosome. The black square nodes indicate reactions; two reactions are linked by a directed edge indicating the reaction flux. The line thickness of each edge is proportional to predicted flux rate change. Source data are provided as a Source Data file.

pool, lipid metabolism, TCA cycle intermediates short of a reduction in glycine levels, the observed reduction in glucose levels is most likely the result of reduced transport across RUPP placenta rather than due to extensive metabolic rewiring ${ }^{69}$.

While this study reports the response of maternal and fetal heart tissues to preeclampsia-driven stress, future studies may help to further evaluate the underlying mechanisms that regulate the effects of preeclampsia on fetal cardiac function. This should include the effects of comorbidities often present with preeclampsia such as obesity and type 2 diabetes. In our RUPP model, we demonstrate that preeclampsia leads to fetal loss, cardiac remodelling and dysfunction showing likely signs of diastolic dysfunction ${ }^{26}$. Furthermore, we demonstrate, for the first time that preeclampsia drives an adaptive metabolic remodelling axis encompassing maternal heart and placenta. However, these adaptations are unable to prevent overall intrauterine growth restriction and fetal loss in RUPP rats despite largely unaltered fetal heart metabolism. We cannot however exclude the possibility that metabolic adaptation of the maternal tissues drives enhanced fetal resorption in order to conserve substrates and $\mathrm{O}_{2}$ for the remaining fetuses. Furthermore, placenta and maternal heart adaptations could be protecting the metabolism of the developing fetal hearts. Nevertheless, long-term consequences of this adaptive maternal cardiovascular changes need to be investigated further.

Potential metabolic mechanisms identified in this study could inform future studies intending to provide better understanding of the increased risk of cardiovascular disease in women affected by preeclampsia. This could also provide improved prevention strategies for reducing the risk of future cardiovascular complications following preeclampsia.

\section{Data availability}

All data supporting the results presented herein are available from the corresponding author upon reasonable request. Cardionet modelling data are provided in a Supplementary File. Database availability: Cardionet MODEL1212040000;

Received: 9 August 2021; Accepted: 31 December 2021

Published online: 21 January 2022 


\section{References}

1. Camm, E. J. et al. Partial contributions of developmental hypoxia and undernutrition to prenatal alterations in somatic growth and cardiovascular structure and function. Am. J. Obstet. Gynecol. 203(495), e424-434. https://doi.org/10.1016/j.ajog.2010.06.046 (2010).

2. Giussani, D. A. et al. Developmental programming of cardiovascular dysfunction by prenatal hypoxia and oxidative stress. PLoS ONE 7, e31017. https://doi.org/10.1371/journal.pone.0031017 (2012).

3. Camm, E. J. et al. Prenatal hypoxia independent of undernutrition promotes molecular markers of insulin resistance in adult offspring. FASEB J. 25, 420-427. https://doi.org/10.1096/fj.10-158188 (2011).

4. Say, L. et al. Global causes of maternal death: a WHO systematic analysis. Lancet Glob. Health 2, e323-333. https://doi.org/10.1016/ S2214-109X(14)70227-X (2014).

5. Mayhew, T. M., Gregson, C. \& Fagan, D. G. Ventricular myocardium in control and growth-retarded human fetuses: growth in different tissue compartments and variation with fetal weight, gestational age, and ventricle size. Hum. Pathol. 30, 655-660. https:// doi.org/10.1016/s0046-8177(99)90090-4 (1999).

6. Veille, J. C., Hanson, R., Sivakoff, M., Hoen, H. \& Ben-Ami, M. Fetal cardiac size in normal, intrauterine growth retarded, and diabetic pregnancies. Am. J. Perinatol. 10, 275-279. https://doi.org/10.1055/s-2007-994739 (1993).

7. Rizzo, G., Capponi, A., Rinaldo, D., Arduini, D. \& Romanini, C. Ventricular ejection force in growth-retarded fetuses. Ultrasound Obstet. Gynecol. 5, 247-255. https://doi.org/10.1046/j.1469-0705.1995.05040247.x (1995).

8. Skilton, M. R., Evans, N., Griffiths, K. A., Harmer, J. A. \& Celermajer, D. S. Aortic wall thickness in newborns with intrauterine growth restriction. Lancet 365, 1484-1486. https://doi.org/10.1016/S0140-6736(05)66419-7 (2005).

9. Roth, H., LeMarquand, G., Henry, A. \& Homer, C. Assessing knowledge gaps of women and healthcare providers concerning cardiovascular risk after hypertensive disorders of pregnancy-a scoping review. Front. Cardiovasc. Med. 6, 178. https://doi.org/10. 3389/fcvm.2019.00178 (2019).

10. Veerbeek, J. H. et al. Cardiovascular disease risk factors after early-onset preeclampsia, late-onset preeclampsia, and pregnancyinduced hypertension. Hypertension 65, 600-606. https://doi.org/10.1161/HYPERTENSIONAHA.114.04850 (2015).

11. Lopez-Campos, G., Bonner, E. \& McClements, L. An integrative biomedical informatics approach to elucidate the similarities between pre-eclampsia and hypertension. Stud. Health Technol. Inf. 264, 988-992. https://doi.org/10.3233/SHTI190372 (2019).

12. Suvakov, S. et al. Overlapping pathogenic signalling pathways and biomarkers in preeclampsia and cardiovascular disease. Pregnancy Hypertens 20, 131-136. https://doi.org/10.1016/j.preghy.2020.03.011 (2020).

13. Burton, G. J., Redman, C. W., Roberts, J. M. \& Moffett, A. Pre-eclampsia: pathophysiology and clinical implications. BMJ 366, 12381. https://doi.org/10.1136/bmj.12381 (2019).

14. Liu, C. M. C. et al. Considerations to model heart disease in women with preeclampsia and cardiovascular disease. Cells https:// doi.org/10.3390/cells10040899 (2021).

15. Alexander, B. T. et al. Reduced uterine perfusion pressure during pregnancy in the rat is associated with increases in arterial pressure and changes in renal nitric oxide. Hypertension 37, 1191-1195. https://doi.org/10.1161/01.hyp.37.4.1191 (2001).

16. Sholook, M. M. et al. Systemic hemodynamic and regional blood flow changes in response to chronic reductions in uterine perfusion pressure in pregnant rats. Am. J. Physiol. Heart Circ. Physiol. 293, H2080-2084. https://doi.org/10.1152/ajpheart.00667.2007 (2007).

17. Gilbert, J. S., Babcock, S. A. \& Granger, J. P. Hypertension produced by reduced uterine perfusion in pregnant rats is associated with increased soluble fms-like tyrosine kinase-1 expression. Hypertension 50, 1142-1147. https://doi.org/10.1161/HYPERTENSI ONAHA.107.096594 (2007).

18. LaMarca, B. B., Bennett, W. A., Alexander, B. T., Cockrell, K. \& Granger, J. P. Hypertension produced by reductions in uterine perfusion in the pregnant rat: role of tumor necrosis factor-alpha. Hypertension 46, 1022-1025. https://doi.org/10.1161/01.HYP. $0000175476.26719 .36(2005)$.

19. Gadonski, G. et al. Hypertension produced by reductions in uterine perfusion in the pregnant rat: role of interleukin 6 . Hypertension 48, 711-716. https://doi.org/10.1161/01.HYP.0000238442.33463.94 (2006).

20. Wallace, K. et al. CD4+ T-helper cells stimulated in response to placental ischemia mediate hypertension during pregnancy. Hypertension 57, 949-955. https://doi.org/10.1161/HYPERTENSIONAHA.110.168344 (2011).

21. Crews, J. K., Herrington, J. N., Granger, J. P. \& Khalil, R. A. Decreased endothelium-dependent vascular relaxation during reduction of uterine perfusion pressure in pregnant rat. Hypertension 35, 367-372. https://doi.org/10.1161/01.hyp.35.1.367 (2000).

22. Sedeek, M. et al. Role of reactive oxygen species in hypertension produced by reduced uterine perfusion in pregnant rats. Am. J. Hypertens 21, 1152-1156. https://doi.org/10.1038/ajh.2008.239 (2008).

23. Bakrania, B. A., Hall, M. E., Shahul, S. \& Granger, J. P. The Reduced Uterine Perfusion Pressure (RUPP) rat model of preeclampsia exhibits impaired systolic function and global longitudinal strain during pregnancy. Pregnancy Hypertens 18, 169-172. https:// doi.org/10.1016/j.preghy.2019.10.001 (2019).

24. Alexander, B. T. Placental insufficiency leads to development of hypertension in growth-restricted offspring. Hypertension 41, 457-462. https://doi.org/10.1161/01.HYP.0000053448.95913.3D (2003).

25. Kupferminc, M. J., Peaceman, A. M., Wigton, T. R., Rehnberg, K. A. \& Socol, M. L. Tumor necrosis factor-alpha is elevated in plasma and amniotic fluid of patients with severe preeclampsia. Am. J. Obstet. Gynecol. 170, 1752-1757 (1994) (discussion 1757-1759).

26. Richards, C. et al. Characterisation of cardiac health in the reduced uterine perfusion pressure model and a $3 \mathrm{D}$ cardiac spheroid model, of preeclampsia. Biol. Sex Differ. 12, 31. https://doi.org/10.1186/s13293-021-00376-1 (2021).

27. Turbeville, H. R. \& Sasser, J. M. Preeclampsia beyond pregnancy: long-term consequences for mother and child. Am. J. Physiol. Renal. Physiol. 318, F1315-F1326. https://doi.org/10.1152/ajprenal.00071.2020 (2020).

28. Faulkes, C. G., Eykyn, T. R. \& Aksentijevic, D. Cardiac metabolomic profile of the naked mole-rat-glycogen to the rescue. Biol. Lett. 15, 20190710. https://doi.org/10.1098/rsbl.2019.0710 (2019).

29. Mansor, L. S. et al. Increased oxidative metabolism following hypoxia in the type 2 diabetic heart, despite normal hypoxia signalling and metabolic adaptation. J. Physiol. 594, 307-320. https://doi.org/10.1113/JP271242 (2016).

30. Chung, Y. L., Leach, M. O. \& Eykyn, T. R. Magnetic resonance spectroscopy to study glycolytic metabolism during autophagy. Methods Enzymol. 588, 133-153. https://doi.org/10.1016/bs.mie.2016.09.078 (2017).

31. Cortassa, S. et al. A computational model integrating electrophysiology, contraction, and mitochondrial bioenergetics in the ventricular myocyte. Biophys. J. 91, 1564-1589. https://doi.org/10.1529/biophysj.105.076174 (2006).

32. Karlstadt, A. et al. CardioNet: a human metabolic network suited for the study of cardiomyocyte metabolism. BMC Syst. Biol. 6, 114. https://doi.org/10.1186/1752-0509-6-114 (2012).

33. Ogata, H. et al. KEGG: kyoto encyclopedia of genes and genomes. Nucleic Acids Res. 27, 29-34 (1999).

34. Aksentijevic, D. et al. Cardiac dysfunction and peri-weaning mortality in malonyl-coenzyme A decarboxylase (MCD) knockout mice as a consequence of restricting substrate plasticity. J. Mol. Cell Cardiol. 75, 76-87. https://doi.org/10.1016/j.yjmcc.2014.07. 008 (2014).

35. Karlstaedt, A. et al. Oncometabolite d-2-hydroxyglutarate impairs alpha-ketoglutarate dehydrogenase and contractile function in rodent heart. Proc. Natl. Acad. Sci. U S A 113, 10436-10441. https://doi.org/10.1073/pnas.1601650113 (2016).

36. Karlstaedt, A., Khanna, R., Thangam, M. \& Taegtmeyer, H. Glucose 6-phosphate accumulates via phosphoglucose isomerase inhibition in heart muscle. Circ. Res. 126, 60-74. https://doi.org/10.1161/CIRCRESAHA.119.315180 (2020). 
37. Aksentijevic, D. et al. Intracellular sodium elevation reprograms cardiac metabolism. Nat. Commun. 11, 4337. https://doi.org/10. 1038/s41467-020-18160-x (2020).

38. Gatford, K. L., Andraweera, P. H., Roberts, C. T. \& Care, A. S. Animal models of preeclampsia: causes, consequences, and interventions. Hypertension 75, 1363-1381. https://doi.org/10.1161/HYPERTENSIONAHA.119.14598 (2020).

39. Tian, X. et al. Effects of placental ischemia are attenuated by 1,25-dihydroxyvitamin D treatment and associated with reduced apoptosis and increased autophagy. DNA Cell Biol. 35, 59-70. https://doi.org/10.1089/dna.2015.2885 (2016).

40. Paauw, N. D. et al. Exposure to placental ischemia impairs postpartum maternal renal and cardiac function in rats. Am. J. Physiol. Regul. Integr. Comp. Physiol. 312, R664-R670. https://doi.org/10.1152/ajpregu.00510.2016 (2017).

41. Irgens, H. U., Reisaeter, L., Irgens, L. M. \& Lie, R. T. Long term mortality of mothers and fathers after pre-eclampsia: population based cohort study. BMJ 323, 1213-1217. https://doi.org/10.1136/bmj.323.7323.1213 (2001).

42. Natale, B. V. et al. Reduced Uteroplacental Perfusion Pressure (RUPP) causes altered trophoblast differentiation and pericyte reduction in the mouse placenta labyrinth. Sci. Rep. 8, 17162. https://doi.org/10.1038/s41598-018-35606-x (2018).

43. Papandreou, I., Cairns, R. A., Fontana, L., Lim, A. L. \& Denko, N. C. HIF-1 mediates adaptation to hypoxia by actively downregulating mitochondrial oxygen consumption. Cell Metab. 3, 187-197. https://doi.org/10.1016/j.cmet.2006.01.012 (2006).

44. Gilbert, J. S., Bauer, A. J., Gingery, A., Banek, C. T. \& Chasson, S. Circulating and utero-placental adaptations to chronic placental ischemia in the rat. Placenta 33, 100-105. https://doi.org/10.1016/j.placenta.2011.11.025 (2012).

45. Saleem, F. R., Chandru, S. \& Biswas, M. Evaluation of total LDH and its isoenzymes as markers in preeclampsia. J. Med. Biochem. 39, 392-398. https://doi.org/10.2478/jomb-2019-0045 (2020).

46. Alqudah, A. et al. FKBPL and SIRT-1 are downregulated by diabetes in pregnancy impacting on angiogenesis and endothelial function. Front. Endocrinol. Lausanne 12, 650328. https://doi.org/10.3389/fendo.2021.650328 (2021).

47. Lopaschuk, G. D., Karwi, Q. G., Tian, R., Wende, A. R. \& Abel, E. D. Cardiac energy metabolism in heart failure. Circ. Res. 128, 1487-1513. https://doi.org/10.1161/CIRCRESAHA.121.318241 (2021).

48. Liu, X. et al. Acetate production from glucose and coupling to mitochondrial metabolism in mammals. Cell 175, 502-513 e513. https://doi.org/10.1016/j.cell.2018.08.040 (2018).

49. Lee, C. F., Caudal, A., Abell, L., Nagana Gowda, G. A. \& Tian, R. Targeting NAD(+) metabolism as interventions for mitochondrial disease. Sci. Rep. 9, 3073. https://doi.org/10.1038/s41598-019-39419-4 (2019).

50. Illsley, N. P., Caniggia, I. \& Zamudio, S. Placental metabolic reprogramming: do changes in the mix of energy-generating substrates modulate fetal growth?. Int. J. Dev. Biol. 54, 409-419. https://doi.org/10.1387/ijdb.082798ni (2010).

51. Zamudio, S. The placenta at high altitude. High Alt. Med. Biol. 4, 171-191. https://doi.org/10.1089/152702903322022785 (2003).

52. Zamudio, S., Baumann, M. U. \& Illsley, N. P. Effects of chronic hypoxia in vivo on the expression of human placental glucose transporters. Placenta 27, 49-55. https://doi.org/10.1016/j.placenta.2004.12.010 (2006).

53. Zamudio, S. et al. Human placental hypoxia-inducible factor-1alpha expression correlates with clinical outcomes in chronic hypoxia in vivo. Am. J. Pathol. 170, 2171-2179. https://doi.org/10.2353/ajpath.2007.061185 (2007).

54. Chakraborty, D., Scott, R. L. \& Soares, M. J. Hypoxia signaling and placental adaptations. Methods Mol. Biol. 1742, 167-183. https:// doi.org/10.1007/978-1-4939-7665-2_15 (2018).

55. Gnarra, J. R. et al. Defective placental vasculogenesis causes embryonic lethality in VHL-deficient mice. Proc. Natl. Acad. Sci. US A 94, 9102-9107. https://doi.org/10.1073/pnas.94.17.9102 (1997).

56. Nuzzo, A. M. et al. Placental adaptation to early-onset hypoxic pregnancy and mitochondria-targeted antioxidant therapy in a rodent model. Am. J. Pathol. 188, 2704-2716. https://doi.org/10.1016/j.ajpath.2018.07.027 (2018).

57. Rana, S., Karumanchi, S. A. \& Lindheimer, M. D. Angiogenic factors in diagnosis, management, and research in preeclampsia. Hypertension 63, 198-202. https://doi.org/10.1161/HYPERTENSIONAHA.113.02293 (2014).

58. Todd, N. et al. Role of a novel angiogenesis FKBPL-CD44 pathway in preeclampsia risk stratification and mesenchymal stem cell treatment. J. Clin. Endocrinol. Metab. 106, 26-41. https://doi.org/10.1210/clinem/dgaa403 (2021).

59. Hooper, S. B. Fetal metabolic responses to hypoxia. Reprod. Fertil. Dev. 7, 527-538. https://doi.org/10.1071/rd9950527 (1995).

60. Hochachka, P. W. \& Lutz, P. L. Mechanism, origin, and evolution of anoxia tolerance in animals. Comp. Biochem. Physiol. B Biochem. Mol. Biol. 130, 435-459. https://doi.org/10.1016/s1096-4959(01)00408-0 (2001).

61. Cannell, G. R. et al. Markers of physical integrity and metabolic viability of the perfused human placental lobule. Clin. Exp. Pharmacol. Physiol. 15, 837-844. https://doi.org/10.1111/j.1440-1681.1988.tb01026.x (1988).

62. Kay, H. H., Zhu, S. \& Tsoi, S. Hypoxia and lactate production in trophoblast cells. Placenta 28, 854-860. https://doi.org/10.1016/j. placenta.2006.11.011 (2007).

63. Baumann, M. U., Zamudio, S. \& Illsley, N. P. Hypoxic upregulation of glucose transporters in BeWo choriocarcinoma cells is mediated by hypoxia-inducible factor-1. Am. J. Physiol. Cell Physiol. 293, C477-485. https://doi.org/10.1152/ajpcell.00075.2007 (2007).

64. Esterman, A. et al. The effect of hypoxia on human trophoblast in culture: morphology, glucose transport and metabolism. Placenta 18, 129-136. https://doi.org/10.1016/s0143-4004(97)90084-9 (1997).

65. Fukasawa, M. et al. Identification and characterization of the hypoxia-responsive element of the human placental 6-phosphofructo2-kinase/fructose-2,6-bisphosphatase gene. J. Biochem. 136, 273-277. https://doi.org/10.1093/jb/mvh137 (2004).

66. Lee, N. Y., Choi, H. M. \& Kang, Y. S. Choline transport via choline transporter-like protein 1 in conditionally immortalized rat syncytiotrophoblast cell lines TR-TBT. Placenta 30, 368-374. https://doi.org/10.1016/j.placenta.2009.01.011 (2009).

67. Kwan, S. T. C. et al. Maternal choline supplementation modulates placental nutrient transport and metabolism in late gestation of mouse pregnancy. J. Nutr. 147, 2083-2092. https://doi.org/10.3945/jn.117.256107 (2017).

68. van den Akker, C. H. et al. Amino acid metabolism in the human fetus at term: leucine, valine, and methionine kinetics. Pediatr. Res. 70, 566-571. https://doi.org/10.1203/PDR.0b013e31823214d1 (2011).

69. Paolini, C. L. et al. Placental transport of leucine, phenylalanine, glycine, and proline in intrauterine growth-restricted pregnancies. J. Clin. Endocrinol. Metab. 86, 5427-5432. https://doi.org/10.1210/jcem.86.11.8036 (2001).

\section{Acknowledgements}

Dr Harold Toms at the School of Biological and Chemical Sciences NMR facility for technical assistance. Sources of funding: School of Biological and Chemical Sciences, Queen Mary University of London, Diabetes UK Grant 19/0005973, Barts Charity Grant MRC 0215, Wellcome Trust 221604/Z/20/Z (DA), British Heart Foundation FS/4YPhD/P/20/34016 (NP), Faculty of Science, University of Technology Sydney, NSW, Australia (LM), Australian Government Research Training Program Scholarship, UTS Research Excellence Scholarship (CR), National Institutes of Health award R00-HL-141702 (AK).

\section{Author contributions}

D.A.- wrote and edited the manuscript, designed the study, performed ${ }^{1} \mathrm{H}$ NMR experiments and analysis, supervised N.P. and interpreted data. N.P. helped to perform ${ }^{1}$ H NMR experiments. C.R. and K.S. performed experiments, data analysis and interpretation. L.M. and K.B. carried out in vivo experiments, supervised C.R. and 
L.M. and performed data analysis and interpretation. A.K. performed in silico analysis (Cardionet), data interpretation and edited the manuscript. All authors contributed to manuscript editing and approved the final draft.

\section{Competing interests}

The authors declare no competing interests.

\section{Additional information}

Supplementary Information The online version contains supplementary material available at https://doi.org/ 10.1038/s41598-022-05120-2.

Correspondence and requests for materials should be addressed to D.A.

Reprints and permissions information is available at www.nature.com/reprints.

Publisher's note Springer Nature remains neutral with regard to jurisdictional claims in published maps and institutional affiliations.

(c) (i) Open Access This article is licensed under a Creative Commons Attribution 4.0 International License, which permits use, sharing, adaptation, distribution and reproduction in any medium or format, as long as you give appropriate credit to the original author(s) and the source, provide a link to the Creative Commons licence, and indicate if changes were made. The images or other third party material in this article are included in the article's Creative Commons licence, unless indicated otherwise in a credit line to the material. If material is not included in the article's Creative Commons licence and your intended use is not permitted by statutory regulation or exceeds the permitted use, you will need to obtain permission directly from the copyright holder. To view a copy of this licence, visit http://creativecommons.org/licenses/by/4.0/.

(C) The Author(s) 2022 